\title{
Proving the case for obstructive sleep apnea (OSA) as a risk factor for cardiovascular disease: the call for a large randomized controlled trial
}

\section{Doug Mcevoy}

Adelaide Institute for Sleep Health, Australia

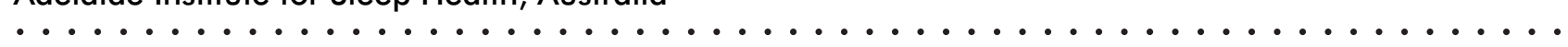

Indian J Sleep Med 2009; 4.3, 77-81

\begin{abstract}
Epidemiological studies in a wide range of ethnic and cultural settings show that obstructive sleep apnea (OSA) (defined as > 15 obstructive events/ hour of sleep) affects about $5-10 \%$ of the population. The rising tide of obesity is almost certainly driving an increase in the prevalence of OSA in emerging economies such as India as well as in more developed countries. An increasing body of evidence over the last 15-20 years suggests that OSA is an independent risk factor for cardiovascular disease. Most of this evidence comes from population or clinic based cohort studies, although there are also a number of short-term randomised controlled trials of OSA treatment showing benefit for intermediate cardiovascular risk markers such as blood pressure, glucose metabolism and arterial vascular reactivity. Also, sudden death and acute myocardial infarction tend to occur preferentially in the nighttime amongst OSA sufferers. While this evidence points strongly to a possible causal link between OSA and cardiovascular diseases such as myocardial infarction and stroke, the international consensus is that definitive evidence for such a link is currently lacking and can only be obtained by rigorously planned and executed large-scale randomised controlled trials. Unfortunately, too often in the past, results from observational data or short term treatment studies have shown "compelling" evidence in favour of a causal relationship between a putative causal factor and cardiovascular disease only to find that properly conducted randomised controlled trials targeting hard endpoints show no such effect and in some instances even harm resulting from the treatment. The Sleep Apnea cardio Vascular Endpoints (SAVE) study is an investigator designed and led multi-centre, international, randomised controlled trial of continuous positive airway pressure (CPAP) treatment plus usual care versus usual care alone in patients with co-occurring OSA and cardiovascular disease, which is designed to help fill this evidence gap. The SAVE trial will extend over 5 years and involve approximately 5000 subjects. It commenced in China and Australia in late 2008 and plans are underway to involve investigators and recruitment sites in India and New Zealand in 2009.
\end{abstract}

Address for correspondence

Doug Mcevoy, Professor

Adelaide Institute for Sleep Health

Repatriation General Hospital

Daw Park, SA, Australia 5041

Email: doug.mcevoy@health.sa.gov.au 


\section{Rationale and Background}

The global burden of cardiovascular disease (CV)

1 t the turn of the century, the World Health Organisation's Global Burden of Disease study ranked coronary artery disease and stroke as the leading causes of premature death and disability worldwide (1). Despite declines in the incidence of these diseases in most developed countries over recent decades the disease burden attributable to cardiovascular disease remains. In developing countries, the burden of $\mathrm{CV}$ disease is rapidly increasing as populations undergo ageing and adopt more Western-oriented lifestyles. The prevention and treatment of CV disease will thus remain a major health priority for all countries. Ongoing campaigns directed at established risk factors such as smoking, dyslipidaemia and hypertension will be important. However, the identification and treatment of new risk factors will also be critically important. Recent evidence points to OSA being a potentially important modifiable risk factor for CV disease.

\section{Obstructive Sleep Apnea}

OSA is a disorder characterised by snoring and repetitive obstructive apnea (complete upper airway collapse) or hypopnea (partial upper airway collapse), hypoxia and sleep disruption. Arterial blood pressure also fluctuates markedly during sleep. OSA, defined as more than 15 apneas and hypopneas per hour of sleep (ie. apnea/ hypopnea index $[\mathrm{AHI}]$ ), affects approximately $10 \%$ of men and $5 \%$ of women (2). As obesity is one of the important causative factors, the prevalence of OSA is projected to increase as rates of obesity rise, worldwide. Studies in India and China indicate that the prevalence of OSA is similar to that in Western countries $(3,4)$. In a landmark study in 1981, Sullivan et al showed that continuous airway positive pressure (CPAP) applied via a nasal mask during sleep could immediately alleviate obstructive apneas and return sleep quality and arterial blood oxygenation to normal. Many subsequent studies have confirmed these findings and shown that excessive daytime sleepiness and neurocognitive dysfunction in those with OSA can be substantially improved by treatment with CPAP. CPAP has thus become the standard treatment for symptomatic moderate-severe OSA and, amongst patients who initially accept this treatment, long-term adherence is around 70-80\% (5), which is similar to other therapies for chronic disorders. While CPAP is a relatively complex medical intervention with high initial cost, its present cost-utility is very favourable in comparison with other common medical interventions (6). If it were proven to be effective in preventing cardiovascular disease as well as daytime sleepiness and accidents its cost-utility would be even greater, placing it in the mainstream of CV disease prevention strategies.

OSA - a potential cause of CV disease

Evidence has accumulated over the last $10-15$ years of a possible causal link between OSA and CV disease. The data can be divided broadly into: (a) population- and clinic-based studies that have compared rates of $\mathrm{CV}$ disease in OSA and non-OSA subjects; (b) descriptive case series or short term comparative interventional studies of CPAP on markers of $\mathrm{CV}$ risk such as $\mathrm{BP}$ (Blood pressure), inflammation and insulin resistance; and (c) studies highlighting the marked nocturnal alterations in $\mathrm{CV}$ physiology that may predispose to sudden ischaemic cardiac or cerebral events. This information has been authoritatively reviewed elsewhere (7) and only key studies will be mentioned here.

Epidemiological evidence Several large cohort studies have examined associations between OSA and CV disease. The Wisconsin Sleep Cohort consists of a random sample of over 3500 men and women aged 3060 years who answered questionnaires in 1988 about snoring and witnessed sleep breathing pauses. One-third of the inception cohort had laboratory sleep studies. Initial cross sectional analyses showed OSA to be an independent risk factor for systemic hypertension (8), and stroke (odds ratio [OR] for AHI>20 compared with $\mathrm{AHI}<5$ was 4.3, 95\% confidence interval [CI] 1.3-14.2) (9). Subsequent longitudinal observations have also shown OSA to predict the development of hypertension (10), stroke (9) and CV mortality (11). The Sleep Heart Health Study is another longitudinal study designed to investigate the relationship between polysomnographically proven sleep apnea and CV disease in over 6000 subjects. After controlling for known confounders, OSA was shown to be a risk factor for hypertension (12), heart failure (4th vs 1st AHI quartiles, relative risk [RR] 2.4 95\% CI 1.2-4.6), stroke (RR $1.61 .0-2.5$ ), and coronary artery disease (RR 1.3 1.0-1.6) (13).

A recent 10-year observational study of 780 male sleep clinic patients and 264 healthy men matched for age and body mass index (BMI) found an increased incidence of fatal (adjusted OR 2.87 95\% CI 1.2-7.5) and non-fatal (OR $3.295 \%$ CI 1.1-7.5) CV events for 
patients with severe untreated OSA (defined as $>30 \mathrm{AHI}$ ) compared with the healthy population (14). Patients who were successfully treated with CPAP had a similar risk of CV death or new events to simple snorers and healthy controls. While these data are strongly suggestive of a causal relationship between OSA and CV disease, the finding with respect to CPAP treatment may be confounded in that CPAP compliant patients may also have been more compliant with $\mathrm{CV}$ medication and lifestyle advice, and received more medical care. In another study of over 1000 sleep clinic patients followed for a median of 3.4 years, the hazard ratio for stroke or death amongst patients with OSA, after controlling for known confounders, was 2.0 (95\% CI 1.1-3.4) (15).

OSA and intermediate $C V$ risk markers There is a strong patho-physiological basis for the observed adverse effects of OSA on CV risk and substantial mechanistic data supporting beneficial effects of CPAP therapy. The strongest evidence relates to the effects of CPAP treatment on blood pressure. A recent meta-analysis of shortduration randomised controlled trials (RCTs) of CPAP in OSA patients concluded, that short-term CPAP significantly lowered BP by approximately $3 \mathrm{~mm} \mathrm{Hg}$ (16). If sustained over the long term a CPAP-induced fall of 3 $\mathrm{mmHg}$ in systolic BP would by itself translate into an 8$10 \%$ reduction in the risk of $\mathrm{CV}$ disease. Data on the long-term effects of CPAP on blood pressure in OSA are lacking at this time. Other studies that compared OSA patients with control subjects or simple snorers have shown increased daytime sympathetic activity, endothelial and vascular dysfunction and increased circulating levels of C-reactive protein (CRP), interleukin 6 and endothelin. Several, although not all, of these markers of CV risk have been shown to improve with CPAP treatment. Increases in metabolic risk factors such as insulin resistance and diabetes have also been reported in OSA although the reports of short-term CPAP treatment effects on glucose control and insulin sensitivity have been inconsistent. A single case-control study has shown an elevation in total cholesterol and decrease in LDL-cholesterol OSA patients. This finding awaits confirmation by other groups. Some studies have also shown increased platelet aggregability, fibrinogen, and blood viscosity in OSA patients although rigid controls for other factors such as medications, caffeine and comorbidities have often been missing. Some but not all studies reported improvements following CPAP. Finally, several studies have reported increased carotid intimamedial wall thickness amongst OSA patients who had no other known CV risk factors, suggesting early atheromatous disease, with one study reporting regression of these changes with CPAP (17).

Nocturnal disturbances in $C V$ physiology and sudden death Acute increases of 10-20 mm Hg in mean systemic and pulmonary artery pressure accompany obstructive apnoeas, and can occur several hundreds of times per night in severe cases. Acute hypoxaemia and sudden sympathetic neural discharge accompany these surges in BP (18). Such sleep-related changes have the potential to increase arterial wall sheer stress, ventricular afterload and cardiac irritability, thus setting the scene for atherosclerotic plaque rupture, myocardial ischaemia and tachyarrhythmias $(19,20)$. Two recent studies found that sudden cardiac death (21) and myocardial infarction (22) amongst those with OSA was two to six times more likely to occur during the hours of sleep rather than in the early rising hours as occurs in the general population. What is not clear from these studies, however, is whether the overall incidence of sudden death and myocardial infarction is increased amongst OSA sufferers. Nevertheless, it appears that obstructive apneas during sleep may predispose to acute coronary thrombosis, malignant arrhythmias, or both.

The need for large, well-designed RCTs of CPAP that target CV endpoints

The data above provide strong support for adverse effects of OSA on CV disease and a real expectation that CPAP treatment might produce reductions in $\mathrm{CV}$ risk. However, the data are not definitive and there are many examples in medicine where non-randomised studies pointed strongly to likely benefit from a particular therapeutic intervention (eg vitamin $\mathrm{E}$ and $\mathrm{CV}$ disease, hormone replacement therapy and $\mathrm{CV}$ disease) only for large-scale RCTs to subsequently show no benefit, or even harm, from treatment (23) As highlighted in recent consensus statements and leading editorials $(7,24)$, the link between OSA, CPAP and CV disease will only be established definitively by conducting well-designed and adequately powered RCTs of OSA therapy. For example, the 2008 American Heart Association/American College of Cardiology Foundation Scientific report "Sleep Apnea and Cardiovascular Disease" (7), having extensively reviewed the current evidence linking OSA and cardiovascular disease concludes that two of the major challenges to be overcome in this field are "widespread comorbidities, including obesity, that obscure a clearer understanding of any independent cardiovascular 
consequence of sleep apnea per se" and "the absence of robust longitudinal interventional studies addressing whether treating sleep apnea confers any tangible benefit in terms of cardiovascular events". The authors believe that the current evidence "should serve as catalyst for definitive intervention studies". It is hoped that the Sleep Apnea cardioVascular Endpoints (SAVE) study, an investigator initiated and led international, multi-centre RCT of CPAP therapy plus usual care versus usual best care alone in high CV risk patients with OSA will provide this evidence, and help resolve the present uncertainty in this field and ensure that the use of CPAP for OSA is evidence-based.

\section{The Sleep Apnea Cardiovascular Endpoints (Save) Study}

The specific aim of the SAVE study is to determine if CPAP treatment will reduce CV events in patients with established coronary artery or cerebrovascular disease and co-existing moderate-severe obstructive sleep apnea (OSA).

Approximately 5,000 patients with co-existing CV disease and moderate-severe OSA will be randomised to CPAP treatment plus standard care versus standard care alone and the incidence of $\mathrm{CV}$ events compared in the two groups over 3-5 years follow-up. The primary endpoint will be a cluster of CV events including CV death, non-fatal myocardial infarction (MI), non-fatal stroke, and hospitalisation for another CV event (unstable angina, transient ischaemic attack [TIA], heart failure).

Key secondary aims are to evaluate the effects of CPAP on

- daytime sleepiness and other sleep apnea symptoms,

- mood, as assessed by the Hospital Anxiety and Depression Scale,

- health-related quality of life (HRQoL), as assessed by the QoL35 questionnaire

SAVE has been in development since late 2004. The protocol is finalised and the organisational structure and feasibility of the study are established. The study is overseen by an Executive Committee of international leaders in sleep and cardiovascular medicine and is being spearheaded by two research institutes in Australia, the Adelaide Institute for Sleep Health and the George Institute for International Health, which have complementary skills in sleep research and large multi- centre cardiovascular trials, respectively. Further details about the trial can be found at www.savetrial.org

Meetings with key opinion leaders and investigators in the participating countries, including in Hyderabad, India, in December 2007, have established that there is a high level of enthusiasm for the study internationally. Site initiation visits and patient recruitment commenced in China and Australia during November and December 2008, respectively. Plans are afoot to enlist and initiate sites in New Zealand and India in 2009. The SAVE study is by far the most ambitious trial in the field of sleep medicine yet attempted. If successful, it will result in an important shift in the way cardiovascular risk is managed by cardiovascular physicians and will provide a new treatment, namely CPAP, in the fight against cardiovascular disease. The principal investigators believe that there is a unique window of opportunity for respiratory/sleep, cardiology and neurology/ stroke clinicians and researchers in the Asian region to combine their expertise to answer this important question and to contribute to an improvement in cardiovascular health, globally.

\section{References}

1. Lopez AD, C.J. L. Murray. The global burden of disease: a comprehensive assessment of mortality and disability from diseases, injuries, and risk factors in 1990 and projected to 2020. Cambridge, MA: Harvard School of Public Health on behalf of the World Health Organization and World Bank, 1996.

2. Young T, et al. Epidemiology of obstructive sleep apnea: a population health perspective. Am J Respir Crit Care Med 2002; 165(9):1217-39.

3. Ip MS, et al. A community study of sleep-disordered breathing in middle-aged Chinese men in Hong Kong. Chest $2001 ; 119(1): 62-9$.

4. Udwadia ZF, et al. Prevalence of sleep-disordered breathing and sleep apnea in middle-aged urban Indian men. Am J Respir Crit Care Med 2004; 169(2):168-73.

5. Collard $\mathbf{P}$, et al. Compliance with nasal CPAP in obstructive sleep apnea patients. Sleep Med Rev 1997; 1(1):33-44.

6. Mar J, et al. The cost-effectiveness of nCPAP treatment in patients with moderate-to-severe obstructive sleep apnoea. Eur Respir J 2003; 21 (3):515-22.

7. Somers VK, et al. Sleep apnea and cardiovascular disease: an American Heart Association/American College of Cardiology Foundation Scientific Statement from the American Heart Association Council for High Blood Pressure Research Professional Education Committee, Council on Clinical Cardiology, Stroke Council, and Council on Cardiovascular Nursing. J Am Coll Cardiol 2008; $52(8): 686-717$. 
8. Young $\mathbf{T}$, et al. Population-based study of sleep-disordered breathing as a risk factor for hypertension. Arch Intern Med 157(15):1997; 1746-52.

9. Arzt $\mathbf{M}$, et al. Association of sleep-disordered breathing and the occurrence of stroke. Am J Respir Crit Care Med 2005; 172(11):1447-51.

10. Peppard PE, et al. Prospective study of the association between sleep-disordered breathing and hypertension. $N$ Engl J Med 2000; 342(19):1378-84.

11. Young $\mathbf{T}$, et al. Sleep disordered breathing and mortality: eighteen-year follow-up of the Wisconsin sleep cohort. Sleep 2008; $31(8): 1071-8$.

12. Nieto FJ, et al. Association of sleep-disordered breathing, sleep apnea, and hypertension in a large community-based study. Sleep Heart Health Study. Jama 2000; 283(14): 1829 36.

13. Shahar E, et al. Sleep-disordered breathing and cardiovascular disease: cross-sectional results of the Sleep Heart Health Study. Am J Respir Crit Care Med 2001; 163(1):19-25.

14. Marin $\mathbf{J M}$, et al. Long-term cardiovascular outcomes in men with obstructive sleep apnoea-hypopnoea with or without treatment with continuous positive airway pressure: an observational study. Lancet 2005; 365(9464):104653.

15. Yaggi HK, et al. Obstructive sleep apnea as a risk factor for stroke and death. N Engl J Med 2005; 353(19):203441 .
16. Bazzano LA, et al. Effect of nocturnal nasal continuous positive airway pressure on blood pressure in obstructive sleep apnea. Hypertension 2007; 50(2):417-23.

17. Drager LF, et al. Effects of continuous positive airway pressure on early signs of atherosclerosis in obstructive sleep apnea. Am J Respir Crit Care Med 2007; 176(7):70612.

18. Somers VK, et al. Sympathetic neural mechanisms in obstructive sleep apnea. J Clin Invest 1995; 96(4):1897904.

19. Peled $\mathbf{N}$, et al. Nocturnal ischemic events in patients with obstructive sleep apnea syndrome and ischemic heart disease: effects of continuous positive air pressure treatment. J Am Coll Cardiol 1999; 34(6):1744-9.

20. Harbison $\mathbf{J}$, et al. Cardiac rhythm disturbances in the obstructive sleep apnea syndrome: effects of nasal continuous positive airway pressure therapy. Chest 2000; $118(3): 591-5$.

21. Gami AS, et al. Day-night pattern of sudden death in obstructive sleep apnea. N Engl J Med 2005; 352(12): 1206-14.

22. Kuniyoshi FH, et al. Day-night variation of acute myocardial infarction in obstructive sleep apnea. J Am Coll Cardiol 2008; $52(5): 343-6$

23. Larkin $M$. Value of HRT in women with heart disease doubted. Lancet 1998; 352(9128):627.

24. Somers VK Sleep-a new cardiovascular frontier. N Engl J Med 2005; 353(19):2070-3. 